\title{
The Intracellular Signalling that Associated with Influenza A Virus Infection
}

\author{
Hany Khalil* \\ Department of Molecular Biology, Genetic Engineering and Biotechnology Research Institute, University of Sadat City, Sadat city, Egypt
}

${ }^{*}$ Corresponding author: Hany Khalil, Department of Molecular Biology, Genetic Engineering and Biotechnology Research Institute, University of Sadat City, Sadat city, Egypt, Tel: +20273291693; E-mail: hkhalil74@gmail.com

Received date: November 22, 2016; Accepted date: February 20, 2017; Published date: February 27, 2017

Citation: Khalil H (2017) The Intracellular Signalling that Associated with Influenza a Virus Infection. Pediatric Infect Dis 2: 38 . doi: $10.21767 / 2573-0282.100038$

Copyright: (C) 2017 Khalil H. This is an open-access article distributed under the terms of the Creative Commons Attribution License, which permits unrestricted use, distribution, and reproduction in any medium, provided the original author and source are credited.

\begin{abstract}
Influenza-A virus infection induces a variety of intracellular signalling pathways that are either antiviral or required to ensure efficient replication. A prominent cellular antiviral event is the activation of cascade that produces type I interferon's (IFNs): IFN- $\alpha$ and IFN- $\beta$. Consequently, an appreciated number of antiviral proteins that encoded by so-called IFN stimulated genes are activated, such as $M \times 1$, PKR and ISG-15 genes. Conversely, virus-supportive signalling processes activated upon infection are I kappa B kinase/nuclear factor kappa-light-chain-enhancer of activated B cells (IKK/ NF-KB), phosphatidylinositol-3kinasee/Akt (PI3K/Akt), and the RNA activating factor-1(Raf-1). Specific inhibition of the latter signalling cascade leads to striking impairment of the replication of all influenza A viruses, as a result of nuclear retention of RNPs in late stages of the replication cycle. In this review, the intracellular signaling that associated with IAV infection will be highlighted to realize the appropriate therapeutically strategy against IAV infection.
\end{abstract}

\section{Keywords: Influenza-A virus infection; Intracellular}

signalling

\section{Introduction}

Influenza virus is a member of the family Orthomyxoviridae and like other orthomyxoviruses, has a single-stranded RNA genome with the negative strand type [1,2]. Influenza represents a major public health threat, causing an estimated 250000 to 500000 deaths per year worldwide. The influenza viruses' dramatic impacts are further compounded by their ability to cause recurring pandemics; the first big pandemic, known as "Spanish Flu" of 1918-1919, caused about 40 million deaths. Many other outbreaks have occurred since that time, though none have been as deadly [3]. Three different types of influenza virus, namely $A, B$, and $C$, have been identified. Together these viruses are antigenically diverse from one to another in comparison of their own viral family Orthomyxoviridae. In most cases of the influenza, especially those that occur in epidemics or pandemics are caused by influenza a virus (IAV), which can affect a variety of animal species. While influenza B virus, which only found in humans, is responsible for many localized outbreaks. The influenza $C$ virus is morphologically and genetically different than the other two viruses and is generally asymptomatic [4]. The most important pathways that are stimulated upon IAV infection include interferon signalling pathway, autophagosome formation and apoptotic signalling cascades. Interferon (IFNs) is a cellular protein produced by lymphocytes and infected cells urgently after several viral and bacterial infections regarding to the innate response to inhibit its replication. Interferon's are classified into three types, IFN type I, II and III according to the receptors and signalling pathway. IFN type I, which includes IFN- $\alpha$, IFN- $\beta$, IFN-K, IFN- $\omega$ and IFN- $\delta$, is known to induce immunity against viral infection [5,6]. IFN type II contains IFN- $\gamma$ which is necessary for immune response to other pathogens more than viruses. IFN type III, which initiates signals through a receptor complex that have a role in antiviral response regulation [7]. Noteworthy, autophagy is originally described as the main catabolic pathway responsible for maintaining intracellular nutritional homeostasis that involves the formation of a unique vacuole, the autophagosome and the interaction with the endosomelysosome pathways $[8,9,10]$. This conserved machinery plays a key role in immuno-protection against different invaders, including pathogenic bacteria, intracellular parasites and some viruses like herpes simplex virus and the tobacco mosaic virus [11]. The autophagy processes is characterized by accumulation of double-membrane cytoplasmic vacuoles regulating degradation events and recycling of cellular contents by delivering cytoplasmic materials, required for degradation, to lysosomes. Moreover, autophagy has been shown to play an important role in cell growth, development, aging and disease pathology $[12,13]$. The activation of PI3K and its downstream effector Akt/protein kinase have been identified at early stage upon influenza infection. Conversely, at late stage of infection, P13K-Akt pathway is inactivated due to the activation of p53 phosphorylation and its natural target p21/waf. Activation of these factors at late stage of infection stimulates apoptotic signalling cascades that facilitate releasing of virus progeny [14] 


\section{IAV Infection Stimulates Interferon Signalling Pathway}

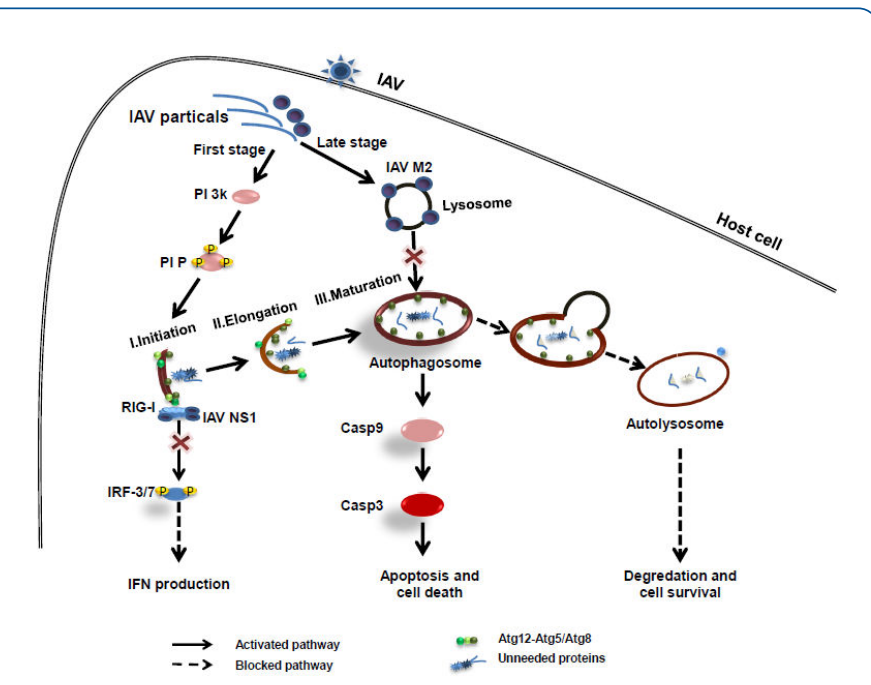

Figure 1: Schematic representation of intracellular signalling that associated with IAV infection: IAV enters the host cells via binding with sialic acids in host cell receptors. Once the virus enters the cells, virus RNA particles stimulate PI 3K-PIP pathway which activate the initiation step of autophagy as well as RIG-I protein kinase. RIG-I proteins is cellular sensor for viral RNA and responsible for stimulation of interferon signaling pathway which includes the activation of interferon regulatory factors $3 / 7$ (IRF-3/7). One of viral strategies to block the interferon pathway is the binding between viral NS1 protein and RIG-I protein using autophagy as a platform for this conjugation. At late stage of infection, viral matrix protein $\mathrm{M} 2$ is targeting cellular lysosomes to inhibit its binding with autophagosomes. Such inhibition is sufficient to induce apoptotic signalling pathway and programmed of cell death to facilitate releasing of virus progeny.

As shown in Figure 1, IAV stimulates the activation of RNA helicase retinoic acid-inducible gene I (RIG-I), the cellular sensor for viral RNA, and both of jun N-terminal kinase (JNK) and activator protein 1 (AP-1) transcription factors [15]. JNK activation is critical in the innate response to IAV infection because JNK-dependent AP-1 factors Trans-activate several antiviral cytokine genes upon infection. Importantly, the AP-1 cooperates with NF-KB and interferon regulatory factors-3,7 (IRF-3,7) to stimulate the expression of type I interferon's [16]. Several studies showed that IAV-NS1 protein is a viral regulator of gene expression that inhibits pre-mRNA processes and nucleocytoplasmic export of cellular mRNAs $[17,18]$. In addition, viral NS1, which has RNA-binding activity, was also shown to inhibit antiviral dsRNA dependent enzymes (RIG-I) and several process including the activation of protein kinase $R$ (PKR), NF-kB, IRF-3 and IRF-7 transcription factors [19]. The IFN type I signalling is mediated through activation of both Janus kinase (JAK) and signal transducers and activator of transcription (STAT) known as JAK-STAT pathway: after receptor activation STAT1/1 homodimers migrate to nucleus and initiate a complex formation with IFN regulatory factor-9 (IRF-9) and interferon stimulated-regulatory elements (ISREs) to induce the expression of many IFN- $\alpha / \beta$ inducible genes [20]. IFNs production, from host cell, is stimulated via several virus infection, some stress condition and endotoxin exposure resulted in enhancement of the cell response by activation of Jun N-terminal kinasee (JNK) and termed stress-activated protein kinasee (SAPK) [21]. Such events theoretically up-regulates the activity of transcription factor of AP-1 family.

\section{IAV Infection Activates Autophagosome Formation}

Several studies have been showed that infection with RNA viruses induced the generation of cytoplasmic vacuoles autophagy, at which viral RNA replication complex accumulates and stimulates viral replication [22]. The autophagy related Atg family such as Atg5, Atg8 (microtubule-associated protein 1 light chain 3, LC3) and Atg12 colocalized with such vacuoles and viral RNA replication complex $[23,24]$. Activation of autophagy machinery is one of the down-stream events of a class III phosphatidylinositl I 3-kinasee (PI3-K) complex. Interestingly, the virus mutant lacking NS1 protein failed to active PI3/Akt indicating that viral NS1 could be responsible for the activation of this pathway [25]. The conjugations between more than 20 Atg proteins have been identified in autophagosome formation recruited from either endoplasmic reticulum (ER) or preautophagosomal structure (PAS). Atg5 and Atg12 are required to form the autophagy vacuoles by recruiting other proteins to autophagosomal membrane from cytosol. The generation of phosphatidylinositol $(3,4,5)$-triphosphate (PIP-3, 4, 5) which recruits additional Atg proteins such as Atg8 (LC3) in conjugation system is essential for autophagosome elongation and maturation (Figure 1). Finally, autophagosome fuses with lysosome containing hydrolytic enzymes that degrade the contents which are recycled for use in protein synthesis and energy production $[26,27,28]$.

Autophagy has been first proposed as a protective mechanism against viral infection by degradation the pathogens in autolysosome leads to decrease viral replication such as the positive stranded RNA tobamoviruses. In contrast, the positive stranded RNA picornaviruses and coronaviruses showed higher replication thereby autophagy formation [22]. Additionally, other viruses, such as poliovirus and mouse hepatitis virus, ensure efficient replication via autophagosomes formation $[29,8,30,31]$. Additionally, other studies showed that autophagy is induced in human single stranded DNA parovirus infected cells which support viral replication [32]. Very recent evidence indicated the essential role of the host autophagy, in enhancing IAV replication in human lung epithelial cells [33,34]. Furthermore, the activation of autophagy is initiated by the conjugation between Atg5-Atg12. Such conjugation negatively regulates IFN- $\beta$ pathway stimulated upon viral infection via direct association with RIG-I and IFN- $\beta$ promoter stimulation 1 (IPS-1) [34-37]. 


\section{Apoptotic Signalling Response}

PI3K/Akt pathway consists of diverse regulatory factors such as p85 and enzymatic subunit ( $p 110$ ) found in several isoforms. Activation of these isoforms reveal both protein kinases and lipid kinases which are known as regulatory factors for various cellular processes such as metabolic regulation, cell growth, proliferation and survival mechanism resulted in subsequent inhibition of caspase 9 and glycogen synthase-kinase 3-B [25]. The activation of PI3K/Akt pathway leads to limitation of virusinduced cell death program or apoptotic process [38] Interestingly, the virus mutant lacking NS1 protein failed to active PI3/Akt indicating that viral NS1 could be responsible for the activation of this pathway [25]. The nuclear accumulation and phosphorylation of p53 pathway, which regulates the apoptosis process, has been shown in low constitutive level upon influenza at early stage of infection, whereas all of its subsequently factors were markedly elevated at late stage of infection leads to apoptosis stimulation [14]. Evidence indicated the essential role of the host autophagy, in enhancing IAV replication in human lung epithelial cells [33]. Other studies on individual influenza virus proteins indicated that the influenza viral $\mathrm{M} 2$ protein is essential to induce the accumulation of autophagosomes. The viral M2 colocalized with autophagosomes and can bind to autophagy-related gene Atg6 (Beclin-1). It is likely that $\mathrm{M} 2$ blocks the formation of autolysosomes through its interaction with Beclin-1, which has been shown to also regulate the fusion between autophagosomes and lysosome [39-40]. The inhibition of autolysosomes formation by $\mathrm{M} 2$ interaction induces programmed cell-death (PCD) resulted in apoptotic signalling in late stage of infection (Figure 1).

\section{Acknowledgments}

Working on viral infection in Dr. Khalil Laboratory is financially support by Science and Technology Development Fund (STDF), Egypt. Through project ID: 4694.

\section{References}

1. Gatherer D (2009) The 2009 H1N1 influenza outbreak in its historical context. J Clin Virol 45: 174-178.

2. Neumann G, Brownlee GG, Fodor E, Kawaoka Y (2004) Orthomyxovirus replication, transcription, and polyadenylation. Biology of Negative Strand Rna Viruses: the Power of Reverse Genetics. Curr Top Microbiol Immunol 283: 121-143.

3. Ghedin E, Sengamalay NA, Shumway M, Zaborsky J, Feldblyum T, et al. (2005). Large-scale sequencing of human influenza reveals the dynamic nature of viral genome evolution. Nature 437: 1162-1166.

4. Van Bressem MF, Van Waerebeek K, Raga JA (1999) A review of virus infections of cetaceans and the potential impact of morbilliviruses, poxviruses and papillomaviruses on host population dynamics. Dis Aquat Organ 38: 53-65.

5. Hsiang TY, Zhao C, Krug RM (2009) Interferon-Induced ISG15 Conjugation Inhibits Influenza A Virus Gene Expression and Replication in Human Cells. J Virol 83: 5971-5977.
6. Rogez C, Martin M, Dereuddre-Bosquet N, Martal J, Dormont D, et al. (2003) Anti-human immunodeficiency virus activity of tau interferon in human macrophages: Involvement of cellular factors and beta-chemokines. J Virol 77: 12914-12920.

7. Levraud JP, Boudinot P, Colin I, Benmansour A, Peyrieras N, et al. (2007) Identification of the zebrafish IFN receptor: Implications for the origin of the vertebrate IFN system. J Immunol 178: 4385-4394.

8. Mizushima N, Klionsky DJ (2007) Protein turnover via autophagy: Implications for metabolism. Annual Review of Nutrition 27: 19-40.

9. Abdelaziz DH, Khalil H, Cormet-Boyaka E, Amer AO (2015) The cooperation between the autophagy machinery and the inflammasome to implement an appropriate innate immune response: do they regulate each other? Immunol Rev 265: 194-204.

10. Yoshimori T, Noda T (2008) Toward unraveling membrane biogenesis in mammalian autophagy. Curr Opin Cell Biol 20: 401-407.

11. Huang J, Klionsky DJ (2007) Autophagy and human disease. Cell Cycle 6: 1837-1849.

12. Yorimitsu T, Klionsky DJ (2005) Autophagy: molecular machinery for self-eating. Cell Death and Differentiation 12: 1542-1552.

13. Khalil H, Tazi M, Caution K, Ahmed A, Kanneganti A, et al. (2016) Aging is associated with hypermethylation of autophagy genes in macrophages. Epigenetics 11: 381-388.

14. Ehrhardt C, Marjuki H, Wolff T, Nurnberg B, Planz O, et al. (2006) Bivalent role of the phosphatidylinositol-3-kinase (PI3K) during influenza virus infection and host cell defence. Cellular Microbiology 8: 1336-1348.

15. Opitz B, Vinzing M, van Laak V, Schmeck B, Heinem G, et al. (2006) Legionella pneumophila induces IFN beta in lung epithelial cells via IPS-1 and IRF3, which also control bacterial replication. J Biol Chem 281: 36173-36179.

16. Ludwig S, Wang XY, Ehrhardt C, Zheng HY, Donelan N, et al. (2002) The influenza $A$ virus NS1 protein inhibits activation of jun Nterminal kinase and AP-1 transcription factors. Journal of Virology 76: 11166-11171.

17. Lu Y, Qian XY, Krug RM (1994) The Influenza-Virus Ns1 Protein - A Novel Inhibitor of Pre-Messenger-Rna Splicing. Genes Dev 8: 1817-1828.

18. Lin DZ, Lan JF, Zhang ZZ (2007) Structure and function of the NS1 protein of influenza A virus. Acta Biochimica et Biophysica Sinica 39: 155-162.

19. Pichlmair A, Schulz O, Tan CP, Naslund TI, Liljestrom P, et al. (2006) RIG-I-mediated antiviral responses to single-stranded RNA bearing 5 '-phosphates. Science 314: 997-1001.

20. Sadler AJ, Williams BRG (2008) Interferon-inducible antiviral effectors. Nature Reviews Immunology 8: 559-568.

21. Yoshida R, Takaesu G, Yoshida H, Okamoto F, Yoshioka T, et al. (2008) TRAF6 and MEKK1 Play a Pivotal Role in the RIG-I-like Helicase Antiviral Pathway. J Biol Chem 283: 36211-36220.

22. Prentice E, Jerome WG, Yoshimori T, Mizushima N, Denison MR (2004) Coronavirus replication complex formation utilizes components of cellular autophagy. J Biol Chem 279: 10136-10141.

23. Fujita M, Sugama S, Nakai M, Takenouchi T, Wei JS, et al. (2007) alpha-synuclein stimulates differentiation of osteosarcoma cells - 
Relevance to down-regulation of proteasome activity. J Biol Chem 282: $5736-5748$.

24. Ozpolat B, Akar U, Mehta K, Lopez-Berestein G (2007) PKC delta and tissue transglutarninase in pancreatic cancer cells are novel inhibitors of autophagy. Autophagy 3: 480-483.

25. Zhirnov OP, Klenk HD (2007) Control of apoptosis in influenza virus-infected cells by up-regulation of Akt and p53 signaling. Apoptosis 12: 1419-1432.

26. Yen WL, Klionsky DJ (2007) Atg27 is a second transmembrane cycling protein. Autophagy 3: 254-256.

27. Tazi MF, Dakhlallah DA, Caution K, Gerber MM, Chang SW, et al. (2016) Elevated Mirc1/Mir17-92 cluster expression negatively regulates autophagy and CFTR (cystic fibrosis transmembrane conductance regulator) function in CF macrophages. Autophagy 12: 2026-2037.

28. Legakis JE, Yen WL, Klionsky DJ (2007) A cycling protein complex required for selective autophagy. Autophagy 3: 422-432.

29. Birmingham CL, Higgins DE, Brumell JH (2008) Avoiding death by autophagy. Autophagy 4: 368-371.

30. Ogawa M, Yoshimori T, Suzuki T, Sagara H, Mizushima $N$, et al. (2005) Escape of intracellular Shigella from autophagy. Science 307: 727-731.

31. Taylor MP, Kirkegaard K (2008) Potential subversion of autophagosomal pathway by picornaviruses. Autophagy 4: 286-289.
32. Nakashima A, Tanaka N, Tamai K, Kyuuma M, IshikawaY, et al. (2006) Survival of parvovirus B19-infected cells by cellular autophagy. Virology 349: 254-263.

33. Zhou Z, Jiang XJ, Liu D, Fan Z, Hu XD, et al. (2009) Autophagy is involved in influenza A virus replication. Autophagy 5: 321-328.

34. Khalil H (2012) Influenza A virus stimulates autophagy to undermine host cell IFN- $\beta$ production. Egyptian Journal of Biochemistry \& Molecular Biology 30: 283-299.

35. Takeshita F, Kobiyama K, Miyawaki A, Jounai N, Okuda K (2008) The non-canonical role of Atg family members as suppressors of innate antiviral immune signaling. Autophagy 4: 67-69.

36. Tal MC, Iwasaki A (2009) Autophagic control of RLR signaling. Autophagy 5: 749-750.

37. Tal MC, Sasai M, Lee HK, Yordy B, Shadel GS, et al. (2009) Absence of autophagy results in reactive oxygen species-dependent amplification of RLR signaling. Proc Natl Acad Sci USA 106: 2770-2775.

38. Garden GA, Budd SL, Tsai E, Hanson L, Kaul M, et al. (2002) Caspase cascades in human immunodeficiency virus-associated neurodegeneration. J Neurosci 22: 4015-4024.

39. Gunnage M, Munz C (2009) Matrix protein 2 of influenza a virus blocks autophagosome fusion with lysosomes. Autophagy 5: 907.

40. Matsunaga K, Saitoh T, Tabata K, Omori H, Satoh T, et al. (2009) Two Beclin 1-binding proteins, Atg14L and Rubicon, reciprocally regulate autophagy at different stages. Nat Cell Biol 11: 385-96. 